\title{
Hiérarchie des relations syntaxiques interprédicationnelles et appropriation en français $L 2$
}

\author{
Manon Buysse \\ Vrije Universiteit Brussel, département TALK \\ manon.buysse@vub.ac.be
}

Le développement de la jonction interpropositionnelle en français L2 constitue un objet de recherche important en linguistique acquisitionnelle. De nombreuses études se sont centrées sur le développement de structures complexes spécifiques : les propositions complétives finies (Diessel \& Tomasello, 2001), les relatives (Giacalone-Ramat, 1999; Hancock \& Kirchmeyer, 2005), les interrogatives "indirectes" (Eckman et al., 1989), les circonstancielles, en particulier les temporelles (Noyau \& Vasseur, 1986 ; Degand \& Hadermann, 2009) ou les causales (Benazzo, 2004).

Ces approches se basent essentiellement sur le développement, éventuellement comparé, des différentes classes de subordonnées distinguées par la grammaire traditionnelle (cf. Welcomme, 2013). Elles ne prennent pas comme point de référence les divers modes de relation interprédicationnelle qui permettent de réaliser la liaison de prédications, et la complexité relative de ceux-ci. Au-delà des divers types de subordonnées, cette contribution part de l'hypothèse que le processus d'appropriation des la jonction interpropositionnelle est déterminé par la complexité syntaxique relative des modes de relations entre prédications. Elle adhère également à l'assomption que l'acquisition progressive de ceux-ci est universelle (Ellis 1989) et ne varie donc pas selon qu'il s'agit de la langue maternelle ou d'une langue seconde. L'étude a donc pour objet de mettre en évidence les aspects structurels de ce processus d'acquisition, indépendamment de l'impact complémentaire de la L1 de l'apprenant.

Pour évaluer la validité de cette hypothèse, nous nous appuierons sur une théorie unifiée de la complexité propositionnelle, celle proposée par la Grammaire du Rôle et de la Référence (Role and Reference Grammar ou RRG ; Van Valin \& LaPolla, 1997 ; Van Valin, 2005). Ce modèle fonctionnaliste, qui accentue à la fois la dimension communicative des systèmes langagiers par une analyse détaillée de productions spontanées, et le caractère universel de leur architecture par une approche typologique des langues, avance un certain nombre d'hypothèses sur le plan du développement de la jonction interpropositionnelle, dont nous essaierons de vérifier la validité pour l'acquisition d'une langue seconde.

\section{Cadre théorique: la Grammaire du Rôle et de la Référence (RRG)}

La RRG prétend proposer un modèle fonctionnel universel permettant de rendre compte de l'interaction entre les composantes syntaxique, sémantique et pragmatique d'une langue donnée. A côté de la Hiérarchie des relations syntaxiques interclausales (Interclausal Syntactic Relations Hierarchy), sur laquelle nous reviendrons plus en détail, elle propose également un continuum sémantique, qui classe la valeur attribuée aux jonctions interprédicationnelles sur la base de l'intensité du lien sémantique entre les prédications, la Hiérarchie des relations sémantiques interclausales (Interclausal Semantic Relations Hierarchy). Enfin, la RRG relie ces deux plans dans un troisième schème général, qui réunit les continuums syntaxique et sémantique, la Hiérarchie des relations interclausales (Interclausal Relations Hierarchy). L'idée principale derrière cette représentation globale est que les liens syntaxiquement les plus forts correspondent généralement aux liens sémantiques les plus étroits, et que la même équivalence entre les deux plans se retrouve au niveau des liens faibles (cf. Van Valin \& LaPolla, 1997 et Van Valin, 2005). 
Concentrons-nous à présent sur la Hiérarchie des relations syntaxiques interprédicationnelles (HRSxI). Elle trouve son point de départ dans une approche stratifiée de la proposition (layered structure of the clause). L'élaboration de celle-ci passe par plusieurs niveaux de complexification syntaxique. Deux prédicats, appelés en RRG des noyaux (nexus), peuvent se combiner afin de former un seul prédicat complexe qui réunit les arguments des deux prédicats originels (exemple 1). Ce niveau de jonction s'appelle la jonction nucléaire :

(1) Lucie a fait tomber son petit frère.

Une autre possibilité est la combinaison de deux cellules (cores), des entités comprenant chacune un prédicat avec ses propres arguments, comme illustré dans l'exemple (2).

(2) Le garçon entend les oiseaux faire du bruit.

Dans l'exemple (1), la séquence a fait tomber constitue un seul prédicat, qui prend comme sujet Lucie et comme objet direct son petit frère. Dans le cas de (2), par contre, les deux prédicats entend et faire sont maintenus sans se fusionner, gardant chacun ses propres arguments (le garçon et les oiseaux pour le premier prédicat; les oiseaux et $d u$ bruit pour le second). Certaines jonctions cellulaires, comme la 'coordination cellulaire' représentée ici, sont caractérisées par le partage d'un ou plusieurs arguments entre les deux prédicats combinés.

Enfin, la complexification de la proposition peut mener à la connexion de prédications à part entière, des clauses, comme dans l'exemple (3), où chacune des deux parties comporte un prédicat avec ses propres arguments et compléments non obligatoires :

(3) Je n'ai pas passé mes examens parce que je n'avais pas étudié.

La RRG distingue en outre trois types de nexus (nexus types). Il s'agit des trois façons différentes de combiner deux structures linguistiques, basées sur deux critères: l'intégration (embeddedness) et la dépendance. Trois modes de connexion sont identifiés. Il y a d'abord la coordination (4) et la subordination (5), des modes de liaison de prédications généralement reconnus dans les grammaires :

J'ai acheté une peinture et je l'ai immédiatement accrochée au mur.

Je pense que Georges est en train d'écrire un roman.

La subordination est caractérisée par une réponse positive aux deux critères distinctifs: l'une des composantes de la proposition (exemple (4) : que Georges est en train d'écrire un roman) est intégrée dans l'autre en tant qu'argument, et de cette façon, elle dépend de la composante matrice et ne pourrait pas fonctionner indépendamment de celle-ci. Cependant, toutes les clauses répertoriées comme subordonnées en RRG ne fonctionnent pas comme argument direct du prédicat; ceci ne vaut que pour le sous-type de 'subordination complément' (com). D'autres cas sont des sous-phrases de type adverbial (ad), donc non obligatoires par rapport à la configuration du prédicat et de ses arguments. On distinguera alors, selon le niveau de jonction où elles se trouvent, la subordination com-cellulaire ou ad-cellulaire, com-clausale ou ad-clausale, (cf. Van Valin \& LaPolla, 1997 ; Van Valin, 2005).

La coordination, par contre, est définie par l'absence d'intégration aussi bien que de dépendance: aucune des deux composantes de la proposition n'est intégrée dans l'autre comme élément indispensable de celleci, et les deux composantes pourraient aussi bien fonctionner comme des propositions à part entière indépendantes. Les notions d'intégration et de dépendance ne s'appliquent donc pas uniquement à la jonction de clauses, mais elles s'étendent aux trois niveaux de jonction distingués par la RRG.

Un troisième mode de connexion est identifié, qui combine l'absence d'intégration avec une forme de dépendance. Cette catégorie de nexus, appelé la cosubordination, est illustrée par l'exemple (6):

Marie a perdu une dent et Louise aussi.

La séquence et Louise aussi constitue une structure qui n'est pas intégrée dans la clause matrice comme argument, mais qui est toutefois dépendante de celle-ci : pour être interprétée, la séquence a besoin de l'information fournie par le prédicat perdu une dent, qui n'est syntaxiquement explicité que dans la 
première clause. Elle ne pourrait donc pas fonctionner indépendamment. Une autre forme de dépendance sans intégration est celle liée à un opérateur partagé (7) :

Jean doit essayer de laver la voiture.

Le verbe modal doit ne porte pas uniquement sur essayer, mais également sur laver; la tentative ainsi que l'action même sont rendues obligatoires par la présence de l'opérateur modal. Cette qualification modale, bien que portant sur les deux cellules prédicatives, n'est exprimée qu'au sein de la première, l'interprétation de la seconde dépendant de l'opérateur présent dans la première.

Cette double tripartition sur le plan des niveaux de jonction ainsi que sur celui des types de nexus débouche sur neuf catégorisations possibles pour les structures syntaxiquement complexes, représentées et illustrées dans le tableau ci-dessous. Pour les langues impliquées dans notre étude, le français et l'anglais, seules sept de ces combinaisons sont autorisées, la jonction nucléaire ne permettant que la cosubordination.

Tableau 1: Types de jonction et de nexus en français

\begin{tabular}{|l|l|l|}
\hline Jonction & Type de nexus & Exemple \\
\hline Nucléaire & Cosubordination & Le chien a fait tomber le bocal. \\
\hline Cellulaire & Cosubordination & Je veux bien t'aider. \\
\hline & Subordination & Fumer est mauvais pour la santé. \\
\hline & Coordination & Le professeur a dit à Lucien de se taire. \\
\hline Clausale & Cosubordination & Michel n'aime pas voyager et moi non plus. \\
\hline & Subordination & Anne ne sait pas qu'elle sera bientôt licenciée. \\
\hline & Coordination & J'aime mes parents, mais je ne les vois pas souvent. \\
\hline
\end{tabular}

Ces sept types de combinaisons syntaxiques peuvent être classés hiérarchiquement, selon la force du lien syntaxique entre les composantes de l'ensemble, sur un continuum appelé la Hiérarchie des relations syntaxiques interprédicationnelles (HRSxI). En ce qui concerne les modes de connexion (types de nexus), la coordination représente le lien syntaxique le moins étroit; contrairement à ce qui est souvent soutenu en linguistique traditionnelle, c'est la cosubordination et non la subordination qui s'y trouve opposée comme relation la plus intense, dans la mesure où l'intégration permet aux prédications subordonnées d'être caractérisées par leurs propres opérateurs et structures argumentales malgré la relation de dépendance, alors que les prédicats ou les prédications cosubordonné(e)s perdent généralement ces attributs importants (opérateurs ou structure argumentale distincts) de leur fonction prédicative. Sur le plan du niveau de jonction, la combinaison de prédicats qui partagent leurs arguments confère aux jonctions nucléaires le statut de connexion intense, tandis que la combinaison de deux clauses entières peut être considéré comme représentant un lien syntaxique nettement plus faible.

Tableau 2: Hiérarchie des relations syntaxiques interclausales (HRSxI)

\begin{tabular}{rc} 
Type de jonction et de nexus & Lien syntaxique \\
\hline Cosubordination nucléaire & FORT \\
Cosubordination cellulaire & $\downarrow$
\end{tabular}




\author{
Subordination cellulaire \\ Coordination cellulaire \\ Cosubordination clausale \\ Subordination clausale \\ Coordination clausale FAIBLE
}

Selon la RRG, l'appropriation de la connexion de prédications se déroulerait à travers une progression de la complexité du niveau de jonction et du mode de connexion (type de nexus), c'est-à-dire selon une hiérarchie des relations syntaxiques pouvant s'instaurer entre les prédications. Le caractère universaliste de la théorie (Van Valin, 2005) implique que la hiérarchie proposée et la séquence de développement en découlant devraient être valides pour l'acquisition de n'importe quelle langue, même si elles sont vérifiées par la RRG dans un contexte d'acquisition d'une L1. L'objectif de notre recherche est donc d'examiner si les principes proposés restent également pertinents pour l'acquisition d'une L2 ou si, au contraire, d'autres facteurs, sans doute spécifiques à la L2, interfèrent.

\title{
2 Méthodologie et questions de recherche
}

2.1. Les données que nous avons analysées proviennent d'un projet plus vaste concernant la production orale en français et en anglais par des élèves de l'enseignement secondaire en Belgique néerlandophone réalisant la même tâche narrative dans les deux langues. Ces deux langues constituent la première et la deuxième langue étrangère du programme scolaire en Flandre. La tâche consiste en la narration, basée sur une bande dessinée sans paroles, des aventures d'un petit garçon à la recherche de sa grenouille domestique qui a disparu (the frog story, "histoire de la grenouille"; Mayer, 1969). Les apprenants ont dû produire la narration en temps réel, ce qui permet d'obtenir une production relativement spontanée en langue étrangère.

Les apprenants du corpus ont été répartis en quatre groupes sur base de mesures globales liées à la correction de la production orale. En particulier, nous avons calculé la proportion de propositions sans erreurs produites par les apprenants, l'adéquation de la conjugaison verbale, et l'emploi correct des prépositions dans la langue cible. Sur base de ces trois mesures, nous avons distingué quatre groupes, dont le premier représente le niveau de maîtrise le plus bas en FL2 et le quatrième le niveau le plus avancé.

Pour cette étude, nous avons analysé le développement de la jonction interprédicationnelle en français dans la production de dix apprenants par groupe. Comme point de référence, nous avons également observé la production orale dans le cadre de la même tâche chez dix locuteurs francophones natifs.

Tableau 3: Aperçu des groupes de locuteurs distingués dans notre étude

\begin{tabular}{|c|c|}
\hline Niveau & Nombre de locuteurs \\
\hline 1 & 10 \\
\hline 2 & 10 \\
\hline 3 & 10 \\
\hline 4 & 10 \\
\hline \hline Natifs & 10 \\
\hline
\end{tabular}


2.2. La hiérarchie des relations syntaxiques interprédicationnelles proposée par la RRG donne lieu à certaines prédictions concernant l'ordre d'acquisition et de développement des structures prédicationnelles syntaxiquement complexes. Cette étude examine si ces prédictions sont confirmées pour l'acquisition du français L2. Nous formulerons celles-ci sous la forme des questions de recherche suivantes :

Q1. Les premiers types de jonction à apparaître dans le processus d'appropriation sont-ils sous-clausaux (donc nucléaires et cellulaires) plutôt que clausaux, et ceci pour les différents types de nexus? En d'autres mots, la cosubordination nucléaire et cellulaire serait produite avant la cosubordination clausale, et la subordination cellulaire se développerait avant la subordination clausale.

L'hypothèse concerne le niveau de jonction :

"Vu que les enfants commencent à produire des constructions de jonction
interprédicationnelle bien avant qu'ils maîtrisent la gamme complète de
constituants et d'opérateurs possibles même en phrases simples, les
jonctions contenant des unités sub-clausales apparaîtront avant celles
contenant des clauses entières." (Van Valin, 2001: 12, ma traduction)

Selon la RRG, il devrait donc être plus facile de produire des structures syntaxiquement complexes sur base de composantes moins élaborées que sur base de clauses entières, même si les premiers niveaux de jonction impliquent un lien syntaxique plus intense que le dernier niveau de jonction.

Q2. Les modes de connexion (types de nexus) non-subordonnés (donc la cosubordination et la coordination) sont-ils employés plus aisément et donc plus précocement que le nexus subordonné au même niveau de jonction? Autrement dit, la cosubordination et la coordination cellulaires seraient utilisées avant la subordination cellulaire, et au niveau de la jonction clausale, les propositions cosubordonnées et coordonnées seraient produites avant les subordonnées, et ceci indépendamment de l'intensité du lien syntaxique interprédicationnel (plus fort dans le cas de la cosubordination et moins fort dans le cas de la coordination).
"En ce qui concerne les types de nexus, la relation juxtapositionnelle, présente en coordination et en cosubordination, est apparemment conceptuellement plus simple que la relation intégrative de la subordination, et par conséquent les premières instances de jonction interprédicationnelle présenteront des types de nexus non subordonnés, c.-à.-d. la coordination et la cosubordination." (Van Valin, 2001: 13, ma traduction)

La cosubordination et la coordination sont deux types de nexus qui n'impliquent pas d'intégration syntaxique, ne demandant pas d'intégrer un élément dans un autre comme argument ou comme participant. La subordination, par contre, impose bien ce genre d'opération syntaxique. Les constructions complexes qui n'imposent pas ce type d'intégration sont censées être plus faciles à conceptualiser et à produire en langue seconde. Ceci rejoint en partie la prédiction classique que la parataxe précède l'hypotaxe (Véronique, 2009).

Q3. Sur les plans des jonctions cellulaires et clausales, la cosubordination est-elle utilisée avant la coordination dans les productions des apprenants?

La RRG pose en principe qu'il devrait être moins difficile dans un contexte de jonction cellulaire de produire une proposition complexe dont les deux composantes sont dépendantes que de produire une proposition dont les deux composantes sont autonomes. 


\begin{abstract}
“À n'importe quel niveau de jonction, les structures cosubordonnées devraient constituer le premier type de nexus non subordonné à surgir: elles sont caractérisées par le partage d'un seul sujet entre les deux composantes, contrairement aux phrases coordonnées, qui ne partagent pas de sujet et qui sont donc syntaxiquement plus difficiles à produire." (Van Valin, 2001: 13, ma traduction)
\end{abstract}

Plus spécifiquement, dans le cas d'une jonction cellulaire cosubordonnée, les prédicats qui sont combinés partagent un sujet, qui n'est exprimé explicitement qu'auprès du premier prédicat. Par contre, la connexion de deux cellules ayant chacune leur propre ensemble d'arguments exigerait plus d'effort des apprenants, et ceci malgré le fait que la cosubordination implique un lien syntaxique interprédicationnel plus fort que la coordination. La même ordre d'apparition devrait être valide au niveau clausal.

\title{
3 Données observées
}

Le tableau 4 représente la proportion des sept types de jonction et de nexus aux quatre niveaux de compétence et dans le groupe des locuteurs natifs, tandis que le tableau 5 fournit chaque fois le nombre moyen de jonctions produites par texte à un certain niveau.

Tableau 4: Proportion de chaque type de jonction et de nexus aux cinq niveaux par rapport au nombre total de jonctions (en pourcentages)

\begin{tabular}{|l|r|r|r|r|r|r|r|r|r|}
\hline & \multicolumn{1}{|c|}{ Nucl } & \multicolumn{4}{|c|}{ Cellulaire } & \multicolumn{4}{|c|}{ clausal } \\
\hline & \multicolumn{1}{c|}{ Cosub } & \multicolumn{1}{c|}{ Cosub } & \multicolumn{1}{c|}{ Sub C } & \multicolumn{1}{c|}{ Sub A } & \multicolumn{1}{c|}{ Coord } & \multicolumn{1}{c|}{ Cosub } & \multicolumn{1}{c|}{ Sub C } & Sub A & Coord \\
\hline 1 & 0,0 & 4,0 & 0,0 & 1,3 & 0,0 & 16,8 & 2,5 & 0,7 & 74,7 \\
\hline 2 & 0,2 & 3,7 & 0,0 & 1,4 & 0,0 & 4,5 & 4,1 & 5,3 & 80,7 \\
\hline 3 & 2,2 & 8,4 & 0,3 & 4,2 & 0,3 & 3,9 & 8,1 & 6,9 & 65,4 \\
\hline 4 & 3,5 & 12,7 & 0,2 & 7,0 & 2,0 & 2,9 & 6,8 & 4,7 & 60,2 \\
\hline \hline Natifs & 10,9 & 11,6 & 0,2 & 4,8 & 1,5 & 12,8 & 5,0 & 10,1 & 43,0 \\
\hline
\end{tabular}

Tableau 5: Nombre total et nombre moyen de jonctions par niveau de compétence

\begin{tabular}{|l|l|l|}
\hline Niveau & Total de jonctions & Moyen par texte \\
\hline 1 & 86 & 8,6 \\
\hline 2 & 240 & 24,0 \\
\hline 3 & 313 & 31,3 \\
\hline 4 & 249 & 24,9 \\
\hline \hline Natifs & 326 & 32,6 \\
\hline
\end{tabular}

\subsection{Niveau 1}

Au niveau le moins avancé, la coordination clausale est clairement le type de combinaison le plus fréquent dans les productions orales des apprenants (74,7\%) (exemple (8)). Elle est suivie par la cosubordination clausale $(16,8 \%)$, représentée majoritairement par des instanciations d'ellipse du sujet 
clausal (exemple (9)). D'autres types de cosubordination clausale, qui ne seraient pas forcément considérés par la tradition comme des cas de coordination, p. ex. Ayant fermé la fenêtre, le garçon quitte la chambre, ne figurent que très rarement dans nos données.

(8) Le chien est à côté un bois et le garçon est ook à côté un bois. $(218, \mathrm{~N} 1)$

(9) Le garçon et le chien regardent la fenêtre et cherchent le grenouille. $(214, \mathrm{~N} 1)$

Les autres types de jonction et de nexus sont rares à ce stade. Tandis que la cosubordination cellulaire et les occurrences du mode de connexion subordonné (cellulaire et clausal) n'atteignent qu'un taux de 4\% au maximum, d'autres catégories ne sont même pas représentées dans les données. Il s'agit plus spécifiquement de la cosubordination nucléaire, de la coordination cellulaire et de la subordination comcellulaire. Cette dernière catégorie est d'ailleurs très rarement représentée dans notre corpus en général. Il s'agit d'un nombre réduit de cas comme Je préfère l'imaginer (Aa27, N3), où une cellule prédicative joue le rôle de participant obligatoire au sein d'une autre cellule prédicative.

\subsection{Niveau 2}

Au deuxième niveau de compétence comme au premier, la coordination clausale est nettement le type de combinaison le plus fréquemment produit $(80,7 \%)$. La cosubordination clausale, par contre, est beaucoup moins productive qu'au premier niveau $(4,5 \%)$, alors que les cas de subordination clausale deviennent nettement plus fréquents. C'est notamment le cas pour la subordination ad-clausale (exemple (10)), ou ce que la théorie traditionnelle appellerait des subordonnées adverbiales ayant un sens autre que temporel ou spatial $^{1}$.

(10) Le petit garçon était un peu fâché parce que le chien tombait. (794, N2)

Les jonctions cellulaires se maintiennent à peu près $(3,7 \% \text { pour la cosubordination (exemple }(11))^{2}$ et $1,4 \%$ pour la subordination ad-cellulaire (exemple (12))) :

(11) Le garçon va courir avec son chien. $(834, \mathrm{~N} 2)$

(12) Quand il était par terre, le bocal se casse alors. (794, N2)

La subordination com-cellulaire et la coordination cellulaire ne sont toujours pas présentes dans les productions des apprenants du niveau 2.

\subsection{Niveau 3}

À partir de ce niveau de compétence, les différentes combinaisons possibles sont représentées, la subordination com-cellulaire (exemple (13)) et la coordination cellulaire (exemple (14)) étant donc produites ici :

(13) Je préfère l'imaginer. (Aa27, N3)

(14) Il dit de ne pas parler à son chien. (Me13, N3)

Si la coordination clausale demeure la catégorie la plus importante $(65,4 \%)$, elle se retrouve néanmoins en net recul. Par contre, la cosubordination cellulaire $(8,4 \%)$ et, dans une moindre mesure, nucléaire progresse. La progression est encore plus nette dans le cas de la subordination cellulaire $(4,5 \%)$ et surtout clausale $(15 \%)$. Dans ce dernier cas, cela vaut autant pour la subordination com-clausale $(8,1 \%)$ (exemple (15)) que ad-clausale $(6,9 \%)$ (exemple (16)). Dans son ensemble, la subordination clausale commence à produire un nombre d'énoncés significatif à partir du troisième niveau. Il en va de même pour la subordination ad-cellulaire (4,2\%). À partir de ce stade, les apprenants produisent donc des propositions plus complexes autant du point de vue du niveau de jonction que du mode de connexion : 
(15) Il pense qu'il entend son grenouille dans un tronc. (M31, N3)

(16) Il se prépare pour le chercher. (Sn25, N3)

La cosubordination nucléaire y est également envisagée, tandis que la subordination com-cellulaire et la coordination cellulaire restent très limitées en fréquence. La cosubordination clausale est en décroissance continue depuis le niveau 1.

\subsection{Niveau 4}

La coordination clausale continue à diminuer en fréquence, mais reste la combinaison la plus fréquente. La cosubordination cellulaire, qui avait déjà progressé au niveau 3, continue à se développer $(12,7 \%$; exemple (17)), ainsi que la subordination ad-cellulaire (7,0\% ; exemple (18)).

(17) La grenouille essaye de s'échapper. (Aa10, N4)

(18) Quand il tombe dans l'eau, il entend une grenouille. (M29, N4)

La réduction de fréquence des jonctions clausales se voit compensée par une tendance chez les apprenants à produire proportionnellement plus de jonctions à des niveaux plus intégrés des prédications.

En conclusion, comme le montre le tableau 4, la grande majorité des modes de connexion présents dans nos données participent à l'origine du niveau de jonction clausal. Les proportions évoluent peu entre le premier et le deuxième niveau. Dès le troisième niveau, avec le développement des compétences des élèves, il y a une forte réduction de la coordination clausale au profit de la subordination clausale et de la cosubordination cellulaire. Le quatrième niveau voit l'accentuation du glissement vers les modes de connexion au niveau cellulaire (taux de $21,8 \%$ pour le groupe de niveau le plus élevé), voire même nucléaire, encore que, dans ce dernier cas, la fréquence globale reste modeste.

\subsection{Niveau d'acquisition en français $\mathrm{L2}$}

Une comparaison entre les productions des apprenants les plus avancés en français L2 et les locuteurs natifs du français impliqués dans cette étude, afin de préciser le niveau atteint par les apprenants dans leur apprentissage du français, mène à certaines constatations intéressantes. Tout d'abord, les locuteurs natifs produisent beaucoup moins de cas de coordination clausale que les apprenants, c'est-à-dire environ deux tiers de ceux produits par les membres du quatrième groupe. Par contre, les locuteurs natifs produisent plus de dix pour cent de cosubordinations aux trois niveaux de jonction, alors que les apprenants en produisent beaucoup moins aux niveaux nucléaire et clausal.

(19) Il se fait encorner par un cerf. (733, natif - cosubordination nucléaire)

(20) Le chien s'amuse avec une ruche et la détache. (737, natif cosubordination clausale)

Ces données générales montrent que les locuteurs natifs disposent toujours de ressources linguistiques nettement plus riches que les apprenants pour connecter les prédications. Elles suggèrent aussi que les apprenants du niveau 4, le groupe le plus avancé dans cette étude, sont loin d'avoir atteint un niveau quasi-natif dans leur processus d'acquisition des modes de connexion interprédicationnelle en français L2.

La haute fréquence de la cosubordination nucléaire (10,9\%) chez les natifs s'explique sans doute par un grand nombre de structures factitives à sens passif joignant faire et un autre prédicat, comme Il s'est fait surprendre par une taupe (733, natif), construction qui ne semble pas encore productive chez les apprenants de notre corpus. 
(21) Il va se faire piquer. (744, natif)

(22) Il se faisait emporter par un cerf. (971, natif)

Une distinction remarquable entre natifs et apprenants concerne la fréquence d'emploi du mode de connexion par ad-subordination aux différents niveaux de jonction. Si les natifs favorisent largement le niveau clausal, les apprenants privilégient par contre le niveau cellulaire. Ce dernier niveau de jonction est d'ailleurs celui où la production des apprenants se rapproche le plus de celle des natifs.

(23) Quand Nicolas va dormir, le grenouille (sic) sort du récipient. (Le20, N4 ad-subordination cellulaire)

(24) Le garçon prend le chien dans ses bras parce qu'il est tout triste. (737, natif - ad-subordination clausale)

Il résulte donc de la comparaison entre apprenants et locuteurs natifs que le niveau le plus avancé dans ce corpus sur le plan de la compétence en français L2 se trouve assez loin d'un usage quasi-natif de cette langue, du moins en ce qui concerne le mode de connexion de prédications. Dans la plupart des cas, le comportement syntaxique des natifs diffère significativement de celui des apprenants du français L2. Il faut donc tenir compte du fait que notre corpus ne couvre pas certaines séquences du processus développemental proposé par la RRG. Il s'agit non seulement des développements ultimes, liés à la productions de locuteurs L2 à la compétence quasi-native, mais aussi des premiers phénomènes de connexion chez les apprenants réellement débutants, situés en-deçà de la maîtrise de notre groupe de niveau 1, qui a déjà reçu deux années d'enseignement de français.

\section{Discussion des résultats}

Dans cette section, nous vérifions si les différentes hypothèses générées à partir de la HRSI sont confirmées par nos données, et nous tentons d'expliquer d'éventuelles divergences.

Q1. Les premières formes de jonction à surgir sont-elles de type sous-clausal (donc nucléaires et cellulaires) plutôt que clausal pour les différents types de nexus ? Ainsi, la cosubordination nucléaire et cellulaire apparaîtrait avant la cosubordination clausale et serait plus fréquemment utilisée par les apprenants débutants; de même, la subordination cellulaire se développerait avant la subordination clausale et connaîtrait également un emploi plus fréquent chez les apprenants des groupes les moins avancés.

Cette première question de recherche sera sous-divisée en trois violets, selon le type de nexus concerné. Ainsi, nous comparerons d'abord la cosubordination sous-clausale avec la cosubordination clausale, ensuite la subordination com-cellulaire avec la clausale, et enfin la subordination ad-cellulaire avec la clausale. Afin de mesurer la différence entre les catégories opposées dans chacun des types de nexus, nous avons calculé chaque fois la proportion des catégories comparées par rapport au total relevé. Ainsi par exemple, pour la première sous-question, nous avons calculé la proportion des cosubordinations nucléaires et cellulaires d'une part, et celle des cosubordinations clausales d'autre part, par rapport à l'ensemble de cosubordinations dans les textes.

Les tableaux 6, 7 et 8 ci-dessous fournissent les moyennes pour chaque groupe d'apprenants et reprennent également la proportion présente dans les productions des locuteurs natifs, le groupe de contrôle. Ces derniers n'ont toutefois pas été inclus dans l'analyse statistique à laquelle nous avons chaque fois soumis les données analysées, c'est-à-dire dans les tests ANOVA que nous avons exécutés dans le programme SPSS. Les résultats des analyses statistiques sont explicités en dessous de chaque tableau. 
Tableau 6 : Proportion des deux sous-catégories de cosubordination en pourcentages

\begin{tabular}{|c|c|c|}
\hline & \multicolumn{2}{|c|}{ Ensemble de cosubordinations (100\%) } \\
\hline Niveau & Nucléaires + cellulaires & Clausales \\
\hline 1 & 23,8 & 76,2 \\
\hline 2 & 53,7 & 46,3 \\
\hline 3 & 76,7 & 23,3 \\
\hline 4 & 89,1 & 10,9 \\
\hline Natifs & 67,6 & 32,4 \\
\hline
\end{tabular}

Nous voyons que la proportion de cosubordinations nucléaires et cellulaires s'accroît par rapport aux cosubordinations clausales, ces dernières étant nettement majoritaires au premier niveau, mais ne représentant plus que $10,9 \%$ dans la production du groupe le plus avancé. Tous les niveaux, pourtant, ne diffèrent pas de façon statistiquement significative l'un de l'autre ; c'est le cas uniquement pour le groupe 1 , qui diffère significativement des groupes $3(\mathrm{p}=0,001)$ et $4(\mathrm{p}=0,000)$, et pour le groupe 2 , qui diffère significativement du groupe $4(\mathrm{p}=0,017)$. Les cosubordinations nucléaires et cellulaires ne semblent donc pas être produites par les apprenants avant les cosubordinations clausales; au contraire, elles représentent une portion limitée de toutes les cosubordinations au premier niveau, et dès le deuxième niveau, la tendance s'inverse. L'avantage en faveur des non clausales devient de plus en plus important au fur et à mesure de développement de la maîtrise du FL2.

La présence immédiate et nombreuse des cas de cosubordination clausale, est sans doute due en partie à la nature orale et spontanée des données recueillies. Comme et ou, dans une moindre mesure, mais sont largement employés comme des marqueurs du cohérence du discours, il n'est pas étonnant que non seulement la coordination (25) mais également la cosubordination clausale (26) figurent très fréquemment dans nos données dès le niveau le moins avancé. Comme mentionné dans la section précédente, dans la plupart des cas de cosubordination clausale dans nos textes, il s'agit de propositions entières à sujet implicite ou élidé.

(25) C'est pendant la nuit et un garçon s'assit devant son lit et il regarde avec son chien à un bocal et dans le bocal il y a un grenouille (sic) et (...). (Me7, N3)

(26) (...) mais le garçon krijgt ... avec le chien et regarder avec un arbre et voit le grenouille et sa copine. $(195, \mathrm{~N} 1)$

Tableau 7 : Proportion des deux sous-catégories de subordination 'com' en pourcentages

\begin{tabular}{|c|c|c|}
\hline & \multicolumn{2}{|c|}{ Ensemble de subordinations 'com' (100\%) } \\
\hline Niveau & Cellulaires (sous-cl.) & Clausales \\
\hline 1 & 0,0 & 100,0 \\
\hline 2 & 0,0 & 100,0 \\
\hline 3 & 2,8 & 97,2 \\
\hline 4 & 1,4 & 98,6 \\
\hline Natifs & 16,7 & 83,3 \\
\hline
\end{tabular}


La proportion des subordinations com-cellulaires par rapport à celles clausales reste toujours très limitée dans les quatre groupes. Son absence totale dans les deux premiers groupes, et sa présence minime dans les deux autres groupes d'apprenants, pourraient être considérées comme indices du développement plus tardif de cette structure parmi les apprenants du français L2. À nouveau, l'hypothèse prédisant le développement précoce des subordonnées sous-clausales avant les clausales semble être infirmée pour ce sous-type de subordonnées. Vu la différence ténue entre les quatre groupes d'apprenants, aucun groupe ne diffère des autres de façon statistiquement significative. Peut-être l'histoire de la grenouille ne donne-telle simplement pas vraiment lieu à des phrases où une cellule sert d'argument au sein d'une autre cellule prédicative, comme dans (27).

(27) Je préfère l'imaginer. (Aa27, N3)

Tableau 8 : Proportion des deux sous-catégories de subordination 'ad' en pourcentages

\begin{tabular}{|c|c|c|}
\hline & \multicolumn{2}{|c|}{ Ensemble de subordinations 'ad' (100\%) } \\
\hline Niveau & Cellulaires (sous-cl.) & Clausales \\
\hline 1 & 50,0 & 50,0 \\
\hline 2 & 12,5 & 87,5 \\
\hline 3 & 42,7 & 57,3 \\
\hline 4 & 56,5 & 43,5 \\
\hline Natifs & 34,8 & 65,2 \\
\hline
\end{tabular}

La répartition des subordonnées 'ad' sur les niveaux de jonction sous-clausale (ou cellulaire, pour ce type de structures) et clausale varie, mais demeure assez proche d'un rapport 50/50 pour la plupart des groupes $\mathrm{d}$ 'apprenants. Uniquement dans les productions du deuxième niveau, le nombre de subordinations adclausales est beaucoup plus important que celui des subordinations ad-cellulaires. En dépit du grand contraste entre la moyenne du groupe 2 et les moyennes des autres groupes, cette différence n'est significative que pour le groupe $4(\mathrm{p}=0,016)$. De nouveau, les données ne semblent pas confirmer l'hypothèse que les subordonnées ad-cellulaires devraient se développer avant les clausales; les deux types de structures sont présents dès le groupe d'apprenants les moins avancés et, en général, maintiennent une répartition assez égale.

En conclusion, les données de l'étude infirment très largement la première hypothèse dégagée par la HRSxI sur le rôle du niveau de jonction dans le processus d'appropriation des modes de connexion interprédicationnelle en acquisition du français L2. C'est exactement le contraire qui se produit : les apprenants produisent d'abord ou beaucoup plus fréquemment les différents modes de connexion au niveau clausal avant de les générer au niveau sous-clausal, niveau qui devient toutefois dominant pour la cosubordination aux stades de maîtrise plus avancés. Le développement des modes de jonction de prédications ne se déroule donc pas selon les prédictions de la HRSI concernant la profondeur du niveau de jonction (la jonction sous-clausale étant plus profonde que la jonction clausale). Les questions de recherche suivantes vérifient l'impact du type de nexus sur l'acquisition des modes de jonctions en français L2.

Q2. Les modes de connexion (types de nexus) non-subordonnés (soit la cosubordination et la coordination) sont-ils employés plus aisément et donc à un niveau plus précoce que la subordination au même niveau de jonction? L'hypothèse porte donc sur le type de nexus : elle pose que la cosubordination ou la coordination cellulaires apparaîtraient avant la subordination cellulaire et seraient produites plus 
fréquemment que cette dernière par les groupes moins avancés. Des assomptions du même ordre sont formulées envers la cosubordination et la coordination clausale par rapport à la subordination clausale.

Les tableaux ci-dessous opposent les types de nexus non subordonnés et subordonnés pour chaque niveau de jonction (le tableau 9 concerne les jonctions cellulaires et le tableau 10 les jonctions clausales).

Tableau 9 : Proportion des jonctions non subordonnées et subordonnées dans l'ensemble des jonctions cellulaires

\begin{tabular}{|c|c|c|}
\hline & \multicolumn{2}{|c|}{ Ensemble de jonctions cellulaires (100\%) } \\
\hline Niveau & $\begin{array}{c}\text { Coordination et } \\
\text { cosubordination }\end{array}$ & $\begin{array}{c}\text { Subordination 'com' } \\
\text { et 'ad' }\end{array}$ \\
\hline 1 & 75,0 & 25,0 \\
\hline 2 & 72,2 & 27,8 \\
\hline 3 & 73,6 & 26,3 \\
\hline 4 & 67,1 & 32,9 \\
\hline Natifs & 73,9 & 26,1 \\
\hline
\end{tabular}

Les jonctions cellulaires non subordonnées, c'est-à-dire les cas de coordination et de cosubordination cellulaire, sont effectivement les structures les plus fréquemment produites dans les différents groupes, mais une baisse proportionnelle de leurs occurrences peut être relevée (leur proportion passe de 3/4 à 1/3 des jonctions cellulaires). Aucune différence n'est cependant statistiquement significative. Ces données semblent donc confirmer l'hypothèse formulée à partir de la HRSxI, c'est-à-dire l'idée que la cosubordination et la coordination cellulaire apparaissent d'abord et sont les plus productives chez les apprenants du niveau le moins avancé.

Soulignons que la coordination cellulaire joue un rôle assez limité dans les productions des apprenants. Ils produisent beaucoup moins de structures comme l'exemple (28), où deux cellules sont considérées comme coordonnées l'une à l'autre, que des cas de cosubordination cellulaire, souvent élicité par des verbes comme vouloir ((29)) et continuer à ((30)).

(28) Il dit à son chien d'être silence (sic). (M16, N3)

(29) Le chien veut regarder dans le bocal. (Le18, N3)

(30) Les deux continuer (sic) à chercher. (M26, N3)

Tableau 10 : Proportion des jonctions non subordonnées et subordonnées dans l'ensemble des jonctions clausales

\begin{tabular}{|c|c|c|}
\hline & \multicolumn{2}{|c|}{ Ensemble de jonctions } \\
\hline Niveau & $\begin{array}{c}\text { Coordination et } \\
\text { cosubordination }\end{array}$ & $\begin{array}{c}\text { Subordination 'com' } \\
\text { et 'ad' }\end{array}$ \\
\hline 1 & 96,3 & 3,7 \\
\hline 2 & 89,8 & 10,2 \\
\hline 3 & 82,5 & 17,5 \\
\hline 4 & 85,0 & 15,0 \\
\hline Natifs & 79,3 & 20,7 \\
\hline
\end{tabular}


En général, les cas de coordination et de cosubordination clausales sont beaucoup plus fréquentes dans les textes étudiés ici que les subordinations au même niveau de jonction. La proportion relative des subordonnées, pourtant, s'accroît nettement du premier aux troisième et quatrième niveaux. Statistiquement, le groupe 1 diffère significativement du groupe $3(p=0,002)$ et du groupe $4(p=0,010)$; les autres contrastes ne sont pas assez grands pour être significatifs.

Il semble vrai ici aussi que les jonctions clausales non subordonnées sont produites en très grands nombres à partir du premier niveau de compétence, et qu'elles sont nettement plus productives que les cas de subordination. Progressivement, la subordination sera de mieux en mieux représentée dans les productions au fur et à mesure que la compétence des apprenants progresse. L'hypothèse concernant le rapport entre non subordonnées et subordonnées basée sur la HRSxI est donc confirmée.

Au niveau de la jonction clausale, c'est essentiellement la coordination clausale qui domine parmi les nexus non subordonnés :

(35) Le chien tombe de fenêtre et le bocal il est kaput. (796, N1)

(36) Ils sont en train de chasser le petit chien, et dans le même moment le garçon regarde dans un trou. (Le18, N3)

Les données sur ce point confortent les observations faites dans le cadre d'autres approches, selon lesquelles la parataxe précède l'hypotaxe.

Même si la subordination clausale croît en importance à partir du deuxième groupe d'apprenants, la catégorie comprenant la cosubordination et la coordination clausale demeure nettement la plus vaste, avec une proportion minimale de $82,5 \%$ des jonctions clausales (niveau 3). La prépondérance de ces deux types de structures est sans doute due en partie à la nature des textes recueillis : il s'agit de narrations orales spontanées, ce qui favorise l'emploi très fréquent de et et, dans une moindre mesure, mais comme des marqueurs discursifs soulignant la poursuite du récit plutôt que comme de véritables connecteurs indiquant la coordination. L'exemple (25), répété comme (37) ci-dessous, illustre cette tendance.

(37) C'est pendant la nuit et un garçon s'assit devant son lit et il regarde avec son chien à un bocal et dans le bocal il y a un grenouille (sic) et (...). (Me7, N3)

Toutes les clauses introduites par et ne devraient donc pas nécessairement être considérées comme des cas de coordination pure ; il peut bien souvent s'agir de cas de coordination descriptive ou additionnelle. Cette tendance vaut aussi bien pour le groupe des locuteurs natifs que pour les quatre groupes d'apprenants, bien que la compétence des premiers en langue maternelle leur permette d'introduire plus de variation dans la façon dont ils marquent la poursuite du récit (p. ex. après, puis, ...) Une autre explication possible est l'influence de la langue maternelle des apprenants, le néerlandais, censé employer souvent une structure coordonnée là où le français ferait usage de la subordination (Guillemin-Flescher, 1981 ; Chuquet et Paillard, 1987). Pourtant, comme les locuteurs natifs du français ne produisent qu'un taux très légèrement inférieur de cosubordonnées et de coordonnées, l'impact réel du transfert du néerlandais L1 nous semble limité.

En conclusion, la deuxième hypothèse de la HRSI est largement confirmée par les données de l'étude. Sur le plan de la jonction sous-clausale, la cosubordination émerge comme type de nexus dominant. Sur le plan de la jonction clausale, c'est le mode de connexion non subordonné en général qui est le plus fréquent, soit les modes qui ne demandent pas d'opération d'intégration. Dans le cas de la cosubordination, il existe bien une forme de dépendance d'une structure sur une autre, mais pas dans le sens d'intégration.

Q3. La cosubordination est-elle utilisée avant la coordination et produite de manière plus fréquente dans les productions des apprenants aux différents niveaux de jonction ? L'hypothèse complète la précédente en ce qui concerne le développement progressif des types de nexus. Elle pose que la cosubordination 
cellulaire apparaîtrait avant la coordination cellulaire et serait produite plus fréquemment que cette dernière par les groupes moins avancés. Des assomptions du même ordre sont formulées envers la cosubordination clausale par rapport à la coordination clausale.

Les tableaux 11 et 12 présentent les données des jonctions non subordonnées en opposant la cosubordination à la coordination aux niveaux de jonction cellulaire et clausal.

Tableau 11 : Proportion des types de nexus cellulaires non subordonnés

\begin{tabular}{|c|c|c|}
\hline & \multicolumn{2}{|c|}{$\begin{array}{r}\text { Ensemble des jonctions cellulaires non } \\
\text { subordonnées }(100 \%)\end{array}$} \\
\hline Niveau & Cosubordination & Coordination \\
\hline 1 & 100,0 & 0,0 \\
\hline 2 & 100,0 & 0,0 \\
\hline 3 & 98,3 & 1,7 \\
\hline 4 & 87,0 & 13,0 \\
\hline Natifs & 89,3 & 10,7 \\
\hline
\end{tabular}

Les données du tableau ci-dessus montrent que la cosubordination cellulaire est présente de manière exclusive dans la production des premiers groupes d'apprenants. Les occurrences de coordination cellulaire n'apparaissent qu'à partir du groupe 3, et ce n'est qu'au niveau le plus avancé que la coordination cellulaire joue un rôle non négligeable en tant que mode de jonction. Statistiquement, le groupe 4 diffère de manière significative des groupes $2(p=0,034)$ et $3(p=0,024)$. L'hypothèse formulée par la RRG s'avère donc vraie pour nos données : la coordination cellulaire devient productive bien plus tardivement que la cosubordination au même niveau de jonction.

Tableau 12 : Proportion des types de nexus clausales non subordonnées

\begin{tabular}{|c|c|c|}
\hline & \multicolumn{2}{|c|}{$\begin{array}{c}\text { Ensemble des jonctions clausales non } \\
\text { subordonnées }(100 \%)\end{array}$} \\
\hline Niveau & Cosubordination & Coordination \\
\hline 1 & 17,9 & 82,1 \\
\hline 2 & 5,1 & 94,9 \\
\hline 3 & 6,0 & 94,0 \\
\hline 4 & 5,2 & 94,8 \\
\hline Natifs & 22,7 & 77,3 \\
\hline
\end{tabular}

Les données indiquent effectivement une présence proportionnellement plus forte de la cosubordination chez les apprenants les moins avancés et une forte baisse de celle-ci en faveur de la coordination chez les apprenants des autres groupes, même si, dans l'ensemble des jonctions clausales non subordonnées, les occurrences de structures coordonnées sont beaucoup plus nombreuses dans la production de tous les groupes d'apprenants. Une différence statistiquement significative apparait entre le groupe 1 d'une part et 
les groupes $2(p=0,043)$ et $4(p=0,046)$ d'autre part. La comparaison avec le troisième groupe est presque significative $(\mathrm{p}=0,059)$.

Comme les cas de coordination clausale sont présentes dans les productions des apprenants dès le niveau le moins avancé de notre corpus, et qu'ils sont beaucoup plus fréquents que les cas de cosubordination au même niveau de jonction, il paraît que l'hypothèse proposée sur base de la HRSxI ne soit pas confirmée. Pourtant, si l'on regarde l'évolution des proportions à travers les différents niveaux de compétence, il est bien vrai que l'importance de la cosubordination diminue tandis que la coordination devient (encore) plus fréquente. Cette tendance semble quand même refléter en partie les prédictions de la HRSxI.

L'hypothèse plus générale formulée à travers la troisième question de recherche peut donc être considérée comme confirmée en grande partie par nos données. Le mode de connexion cosubordonné est en effet proportionnellement plus productif pour tous les niveaux de jonction aux stades initiaux de notre corpus pour perdre ensuite en importance au profit de la coordination, même si la proportion d'occurrences entre les deux types de nexus est très variable selon le niveau de jonction, ce qui semble indiquer que ce dernier joue également un rôle dans la rapidité et l'ampleur du processus.

\section{Conclusions}

Nous pouvons conclure de cet examen que les prédictions de la RRG sur le plan de l'acquisition des liens interprédicationnels syntaxiques sont partiellement confirmées pour l'acquisition du français comme langue seconde.

La première hypothèse, concernant le niveau de jonction, n'est pas confirmée par nos données. Elle postulait que les jonctions de niveau sous-clausal (c'est-à-dire nucléaires et cellulaires) seraient acquises et employées productivement avant les jonctions clausales. Contrairement aux prédictions, ce sont les relations clausales qui sont produites en très grands nombres dès le niveau de compétence le moins avancé, le niveau sous-clausal ne devenant proéminent que dans un stade de développement plus tardif chez les apprenants L2 de notre corpus.

Les deux autres hypothèses, par contre, concernant les modes de connexion, sont bien confirmées en grande partie par les productions des apprenants de notre corpus. En premier lieu, nous avons vérifié si les jonctions non subordonnées apparaissent plus fréquemment dans les stades précoces du parcours développemental des apprenants. La coordination et la cosubordination cellulaires et clausales sont effectivement plus productives au début de leur acquisition en L2 que la subordination, ce qui corrobore l'hypothèse pour les productions analysés dans le contexte de cette contribution.

La dernière hypothèse postulait que, parmi les nexus non subordonnés, la cosubordination est plus fréquente à un niveau précoce chez les apprenants L2 que la coordination. Nos données la confirment au niveau cellulaire comme clausal. Même si les cas de coordination clausale sont beaucoup plus fréquents que les cas de cosubordination clausale dès le premier groupe de notre corpus, nous pouvons cependant constater une nette décroissance des cas de cosubordination. Cette prédominance générale des cas de coordination clausale pourrait s'expliquer en partie par de nombreuses occurrences de «fausse coordination », marquant plutôt la continuité discursive, liées au type de tâche (orale et spontanée) et en partie aussi peut-être à la langue maternelle des apprenants. Elle pourrait enfin être liée à l'absence du stade quasi natif dans notre corpus, où certains développements non représentés dans nos données pourraient encore avoir lieu.

Les prédictions concernant l'influence du type de nexus, contrastant en premier lieu les deux modes non subordonnés et la subordination, et en deuxième lieu la coordination et la cosubordination, sont largement confirmées, tandis que ce n'est pas le cas pour la première prédiction, concernant l'influence du niveau de 
jonction (le rapport entre les jonctions sous-clausales, donc nucléaires et cellulaires, et les jonctions clausales). Le paramètre du niveau de jonction semble donc être un paramètre moins important pour le processus d'acquisition de la jonction interprédicationnelle en L2 que le mode de connexion exploité. Les observations à propos de la troisième question de recherche ont pourtant relevé des tendances partiellement contradictoires dans l'évolution comparée des nexus cosubordonnant et coordonnant entre les niveaux cellulaire et clausal. Il faudra donc soumettre ce paramètre à une analyse plus approfondie dans la continuation de notre recherche. Cependant, à première vue, l'impact du niveau de jonction semble lié au type de nexus et ne paraît pas jouer indépendamment du mode de connexion.

Notons pour terminer que cette contribution ne tient compte que du côté syntaxique de la jonction interprédicationnelle, alors que c'est l'interaction syntaxe-sémantique qui est fondamentale pour l'approche de la RRG. Néanmoins, cet article montre déjà que les hypothèses basées sur la HRSxI ouvrent des perspectives stimulantes pour mieux saisir le développement des liens interprédicationnels en langue seconde.

\section{Références bibliographiques}

Benazzo, S. (2004). L'expression de la causalité dans le discours narratif en français L1 et L2. In: Langages, 155, pp. 33-50.

Chuquet, H. \& M. Paillard. (1987). Approche linguistique des problèmes de traduction : anglais-français. Paris : Ophrys.

Degand, L. \& P. Hadermann. (2009). Structure narrative et connecteurs temporels en français langue seconde. In : Havu, E., Härmä, M., Helkkula, M., Larjavaara, M, \& U. Tuomarla (éds.), La langue en contexte. Actes du colloque "Représentations $d u$ sens linguistique $I V »$, Helsinki 28-30 mai 2008. Helsinki: Société Néophilologique, pp. 19-34.

Diessel, H. \& M. Tomasello. (2001). The acquisition of finite complement clauses in English: A corpus-based analysis. In: Cognitive Linguistics, 12, pp. 1-45.

Dixon, R. M. W. (1972). The Dyirbal language of north Queensland. Cambridge: Cambridge University Press.

Eckman, F., Moravcsik, E., \& J. Wirth. (1989). Implicational universals and interrogative structures in the interlanguage of ESL learners. In: Language Learning, 39, pp. 173-205.

Ellis, R. (éd.). (1989). Classroom language learning. Edition spéciale de System, 17, 2.

Gass, S. M. \& L. Selinker. (1983). Language transfer in language learning. Rowley : Newbury House Publishers.

Giacalone Ramat, A. (1999). Functional typology and strategies of clause connection in second-language acquisition. In: Linguistics 37,3: pp. 519-548.

Guillemin-Flescher, J. (1981). Syntaxe comparée du français et de l'anglais : Problèmes de traduction. Paris : Ophrys.

Hancock, V. \& N. Kirchmeyer. (2005). Discourse structuring in Advanced L2 French: The relative clause. In: Dewaele J.-M. (éd.), Focus on French as a Foreign Language. Clevedon : Multilingual Matters, pp. 17-35.

Mayer, M. (1969). Frog, where are you? New York: Dial Press.

Noyau, C. \& M.-T. Vasseur. (1986). L'acquisition des moyens de la référence temporelle en français langue étrangère chez des adultes hispanophones. In : Langages, 21 (84), pp. 105-117.

Odlin, T. (1989). Language transfer: Cross-linguistic influence in language learning. Cambridge: Cambridge University Press.

Van Valin, R. (2001). The acquisition of complex sentences: a case study in the role of theory in the study of language development. In: Boyle, J., Lee, J.-Y. \& A. Okrent. Chicago Linguistic Society Parasession, 36, pp. 511-531. 
Van Valin, R. (2005). Exploring the syntax-semantics interface. Cambridge: Cambridge University Press.

Van Valin, R. \& R. J. LaPolla. (1997). Syntax: Structure, meaning and function. Cambridge: Cambridge University Press.

Véronique, D. (éd.) (2009). L'acquisition de la grammaire du français langue étrangère. Paris : Didier.

Welcomme, A. (2012). La jonction interpropositionnelle à l'oral et à l'écrit en français langue étrangère. Thèse de doctorat, Vrije Universiteit Brussel.

Wilkins, D. P. (1989). Mparntwe Arrernte (Aranda): Studies in the structure and semantics of grammar. Thèse de doctorat, Australian National University.

${ }^{1}$ Les adverbiales temporelles et spatiales se voient classées en RRG comme des cas de subordination adcellulaire, vu qu'elles ont un impact immédiat sur la cellule et non sur la proposition entière.

${ }^{2} v a$ et courir, dans cet exemple, sont considérés comme des prédicats ayant chacun leur propre ensemble d'arguments; selon la RRG, il s'agit donc d'un cas de cosubordination cellulaire. Pour plus d'informations, voyez Van Valin 2005. 